\title{
The Research on Electronic Data Backup and Recovery System Based on Network
}

\author{
Wei XU ${ }^{1, a}$, Juan LUO ${ }^{2}$ \\ ${ }^{1}$ JiangXi People's Police College, Nanchang 330103,China \\ axuwei923@yeah.net
}

Keywords: Electronic Data Backup; Data Recovery; Network

\begin{abstract}
With the widely application of the computer system and the continuous development of information technology, the rapid increase of data quantity also in these data has become an indispensable factor in our work and life; To transfer the data resources, processing, analysis, processing, thus constituted a variety of application system, provide convenience for our production and living and service. In this paper, based on the realization of the network data backup and restore adopted by the customer, the management, server layer composed of system structure. Through the system can realize the network data management and data backup and restore automatically.
\end{abstract}

\section{Introduction}

As high-tech products represented by computer and network technology rapid development and extensive application of information security is increasingly attached great importance to by the people. Data in the computer is the most precious wealth, computer hardware and software to perform various operations, in essence is to use the data resource [1]. Especially the high-speed Internet application development, cloud computing, cloud storage technology gradually popularization of today, carrying tend to rely on a single system application dozens or even thousands of servers running together, application system to produce the large data capacity, distribution in diversification. In this case, how to ensure that distributed efficient application of large capacity data backup and disaster occurred in a timely manner to implement data recovery, ensure the consistency of disaster recovery on all computers, become a new hot topic in the field of data security [2].

This paper analyzes the classification of the data backup technology and the main method of data backup and recovery, analyzed the main methods of network data storage and data backup, choose the way this system use, based on the realization of network data backup and restore adopted by the customer, the management, server layer composed of system structure. Through the system can realize the network data management and data backup and restore automatically

\section{Data backup and recovery requirements analysis}

According to the backup and recovery technology is essentially based on management plan, will build a copy of the important data, to save a copy of the data to different storage location to the original data, when the original data loss or damage, according to the strategy through copy according to the data recovery process of the original data. The data backup is the precondition of data recovery, data recovery is the ultimate goal of data backup, and two processes work together finally to ensure the safety of data storage [3].

At present, the computer system to return to work still exists many problems and difficulties. First of all, general computer users than ordinary computer system using personnel, for the professional knowledge of the computer system is not clear, cannot correctly grasp the working principle of the computer system can't effective backup and data processing system of fault. Second, although specific unit has the ability of system recovery, due to the confidentiality requirements, computer systems are often not able to send to other units. In addition, because at present a lot of popular computer application system, its location is scattered distribution state, when the problem 
occurs, technical support staff can't timely solve the problems and brings great inconvenience to the work.

Facing various risks in the era of network data information, data security is very important, and the data backup and recovery is a powerful guarantee of data security. Traditional local data backup and recovery technology can avoid the local due to software and hardware failures, man-made wrong operation and illegal procedures (including viruses), and other damage [4]. When faced with serious destruction of the local system, suitable for local single way of traditional data backup can't satisfy the requirement of the user network service system backup, you need to use remote network data backup and recovery technology. A perfect network data backup system in addition to include data backup software and hardware level physical fault tolerance level, should be able to the relationship between the backup data.

\section{Backup and restore and related technology}

Backup to the object under the condition of damage or loss to restore some of objects stored, this provides the recovery process of information and data you need. Data backup objects is the data in information system, is to prevent these data because of natural disasters, hardware failure, software error, loss or damage of man-made wrong operation can be resumed [5]. The data backup is based on data recovery, for the purpose of the data backup is greatly reduced the risk of data storage system. According to different backup recovery goals developed all kinds of backup recovery technique, common backup recovery technique is described below.

Distributed backup and restore. When the server distribution location relatively decentralized, and are not able to or unnecessarily expensive SAN system. There are important data service we can use in each local increase storage devices directly connect to the server. On the server to deploy the corresponding storage management software, the execution of corresponding storage strategy. This way is relatively simple, fast deployment of implementation, but the downside is not very good implementation of sharing data in a larger scope. A distributed backup system structure is shown in figure 1.

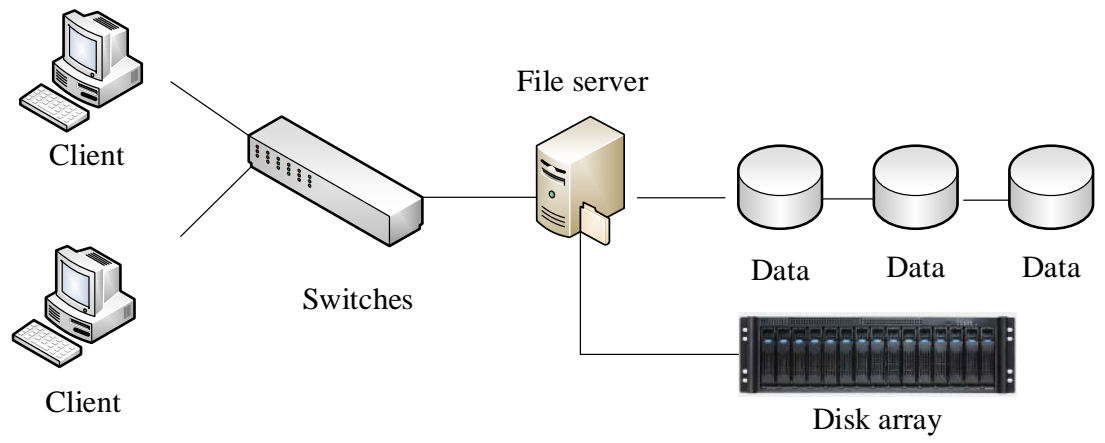

Figure 1. Distributed backup system structure

Network backup and recovery based on the storage area. The network backup solution based on the storage area, the "independent of LAN" and "server" independent two application modes. Based on the storage area network backup structure as shown in figure 2. "LAN" independent backup and recovery is the application server through the optical fiber storage network directly connected storage devices, storage space by LUN transparent assigned to the server. Application server storage data do not take up any network bandwidth, does not affect the application of the transmission of the data stream. The actual backup data only, in SAN domain for the application server, it is used directly with backup mechanism to protect the storage space. 


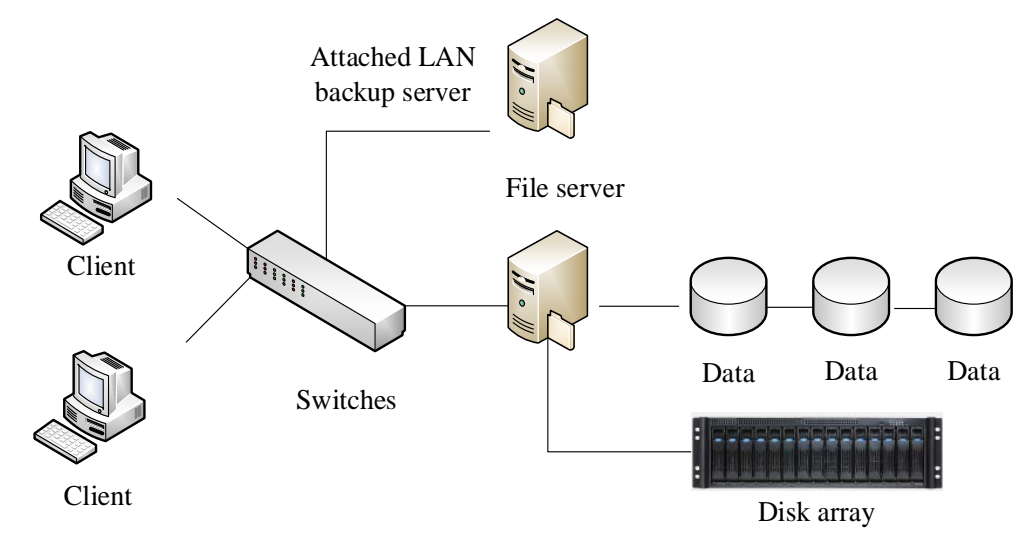

Figure 2. Structure of network backup and recovery based on the storage area

\section{Electronic data backup and recovery system based on the network}

In this paper, network backup and restore system is built on the backup structure. By three layers of organization structure in this paper, system design, the client layer, service layer, the management, on the basis of the corresponding hardware and storage equipment, through the data storage management software, the network of centralized management of data backup and recovery. System realizes the automation of data backup, automatic file archiving, data storage and data recovery, and other functions. This system not only has the basic function of the network data backup, network system also USES PKI security system, through the system of digital certificates, level 1 certification level, the level of trust level, set up a trust chain, identification of all the devices in the network, so that we can ensure the security of the whole system.

Network data backup system based on hierarchical structure is designed, including client layer, service layer, and the management of three parts. Customer layer is a system to manage customer computer resources; Management is the core of the system, including management, backup, backup adapter console and certification center; the service layer provides storage server resources. System architecture as shown in figure 3.

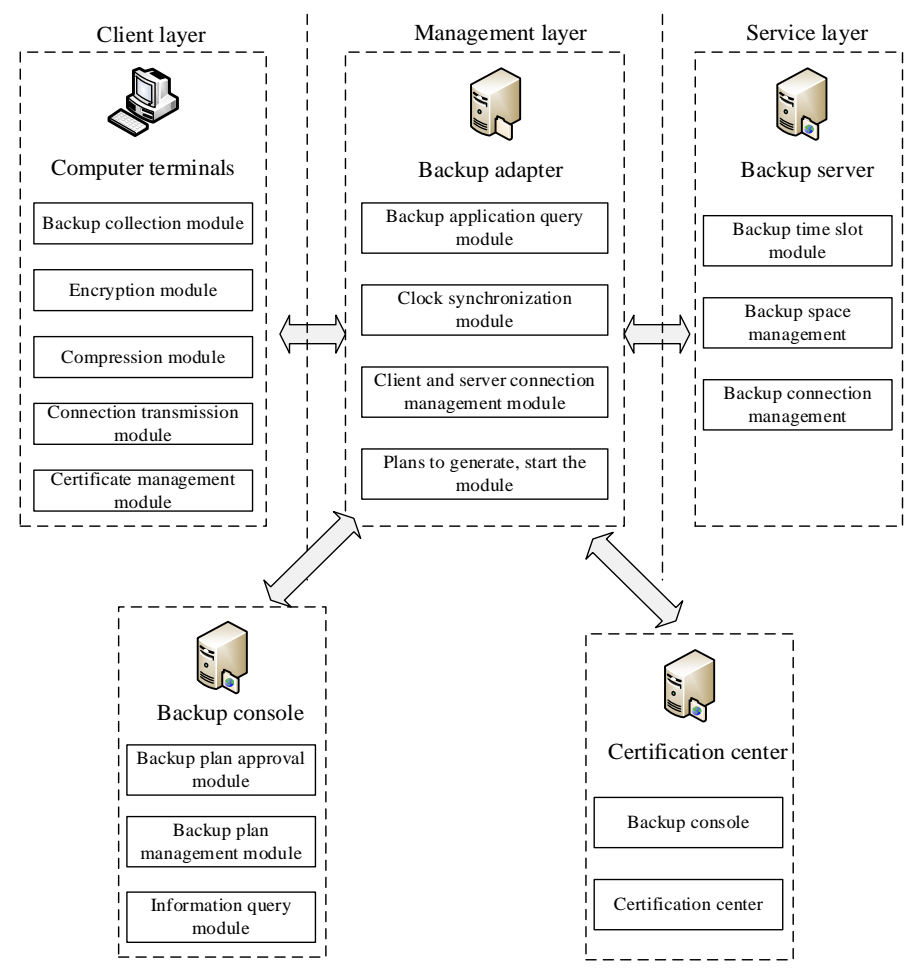

Figure 3. Structure of Electronic data backup and recovery system based on the network 


\section{Conclusion}

With the development and application of cloud computing technology, under the network environment for data distributed storage has become an important developing direction of backup technology. Designed in this paper based on the network data backup and restore system, can be solved by a few hardware to spread the centralized backup file, at the same time provide a convenient recovery mechanism. System fully consider the entire backup recovery process, the client and the safety of the backup objects. Thirty percent through customer layer, service layer, the management organization, user authentication, user management, data compression, data encryption, decryption, data backup, recovery management, etc. to form an organic whole system.

\section{Acknowledgements}

The project was supported by 2013 Scientific Research Fund of Jiangxi Provincial Education Department of China (No.12766)

\section{References}

[1] Shah J, Rajgor D, Pradhan S, et al. Electronic data capture for registries and clinical trials in orthopaedic surgery: open source versus commercial systems[J]. Clinical Orthopaedics and Related Research ${ }^{\circledR}, 2010,468(10):$ 2664-2671.

[2] Xiao K, Xiao D, Luo X. Smart water-saving irrigation system in precision agriculture based on wireless sensor network[J]. Transactions of the Chinese Society of Agricultural Engineering, 2010, 26(11): 170-175.

[3] Meyer J, Ostrzinski S, Fredrich D, et al. Efficient data management in a large-scale epidemiology research project[J]. Computer methods and programs in biomedicine, 2012, 107(3): 425-435.

[4] Prahlad A, Schwartz J. Systems and methods for performing storage operations using network attached storage: U.S. Patent 7,546,324[P]. 2009-6-9.

[5] Gokhale P, Lu J, Lu Y, et al. Systems and methods for recovering electronic information from a storage medium: U.S. Patent 7,873,802[P]. 2011-1-18. 